\title{
Are Perceived Comparative Risks Realistic among High-Risk Sports Participants?
}

\author{
Cécile Martha' and Jason Laurendeau ${ }^{2}$ \\ ' University of the Mediterranean, Aix-Marseille II, Marseilles, France \\ ${ }^{2}$ University of Lethbridge, Lethbridge, Alberta, Conada
}

\begin{abstract}
This paper examined how risk sports practitioners, compared with those of the average sports participant, perceive their abilities to manage risks (AMR) and their vulnerability to a serious injury (VSI) whilst participating. We also examined which variables influence perceived comparative VSI. High-risk and moderate-risk sports participants ( $n=432$ ) completed measures of perceived personal AMR, perceived comparative AMR and VSI, and motive of playing to the limit. Results showed that high-risk sports practitioners perceived their VSI as being higher than the average sports participant, while moderate-risk practitioners perceived their VSI as being lower. Perceived comparative VSI was negatively related to perceived personal AMR and positively related to past injury episode, sporting experience, and playing to the limit. In conclusion, perceived comparative risks were similarly realistic amongst high-risk sports practitioners. Future research is needed to further examine the role that perceived comparative risks play in the risk-taking decision-making process.
\end{abstract}

Key words: comparative optimism, realism, perceived abilities, playing to the limit, past injury episodes

In a culture in which taking unnecessary risks is often seen as foolish, senseless, and even reckless (Lupton, 1999), there seems to be something of a trend toward the increasing acceptance of risk in recreational activities. In the field of sport participation, there is considerable evidence that risk, rather than something to be avoided, is constitutive of many sporting experiences (Celsi, Rose, \& Leigh, 1993; Donnelly, 2004; Kusz, 2004; Lyng, 1990; Young, 1993). Lyng's (1990, 2005) notion of edgework conceptualizes voluntary risk taking as exploring the limits of one's ability and/or the technology one is using while maintaining enough control to successfully negotiate the boundary between chaos and order (Lyng, 1990). Crowding the edge (Lyng, 1990), or playing to the limit (Griffet, 1994), involves taking progressively greater risks in the activity, such as jumping 
from lower or more technical objects in BASE-jumping (Martha \& Griffet, 2006) or executing a hook turn in skydiving (Laurendeau, 2006). In the literature on the rationale for such edgework experiences, the sensation-seeking trait, defined as the "seeking of varied, novel, complex, and intense experiences" (Zuckerman, 1994, p. 27), has been widely investigated. Numerous outdoor sports and activities have been found to attract individuals who rate high in sensation seeking (Breivik, 1996; Rossi \& Cereatti, 1993). The need for arousal, thrill, and adventure may go some way toward explaining why high sensation seekers engage in high-risk sport or take greater risks whilst participating in the same sport, such as in rock climbing or kayaking, than participants who rate low in sensation seeking (Slanger \& Rudestam, 1997).

However, Zuckerman's sensation-seeking model does not tell us how high-risk sportspersons perceive themselves to be exposed to the risk of injury or even death whilst participating. Social psychologists and sociologists have investigated participants' perceived vulnerability, as well as their self-efficacy (Bandura, 1997) in the presence of the risk; that is, their confidence in their perceived abilities to manage risk. Studies have shown that, despite evidence of hazard in their sports, participants in such activities as mountaineering (Delle Fave, Bassi, \& Massimini, 2003; Demirhan, 2005), rock climbing (Llewellyn \& Sanchez, 2008; Llewellyn, Sanchez, Asghar, \& Jones, 2008), skydiving (Laurendeau, 2006; Moen \& Rundmo, 2005), kayaking (Slanger \& Rudestam, 1997), and adventure racing (Schneider, Butryn, Furst, \& Masucci, 2007) trust themselves to negotiate risky situations and believe in their abilities to cope with risk. These studies, however, have not considered whether participants express a comparative optimism, the perception that they are at lower risk of getting injured or are better able to manage risks than their peers (Harris \& Middleton, 1994; Shepperd, Carroll, Grace, \& Meredith, 2002). There exist ethnographic-based studies which have suggested that high-risk sportspersons might deny their vulnerability by comparing themselves to other athletes (Donnelly, 2004; Laurendeau, 2006; Schneider et al., 2007). For instance, skydivers tended to attribute casualties of accidents to others not possessing "the right stuff" (Lyng, 1990, p. 859) or to poor judgements peers make regarding safety (Laurendeau, 2006, p. 596). According to Donnelly (2004), using social comparison allows sportspersons not only to believe that they are physically safe within their own perception of risk but to intensify their feeling of success as they negotiate risk as well.

There exist few quantitative studies that have examined high-risk sports participants' risk perception from a social comparison perspective. Moreover, existing sports studies have reported contradictory results: High-risk sportspersons, such as rock climbers, assessed their vulnerability of getting seriously injured either similarly or higher than their peers (Martha, Sanchez, \& Gomà-i-Freixanet, 2009), but the inverse comparative assessment has been observed. Indeed, Moen and Rundmo (2005) have shown that skydivers expressed a comparative optimism since they assessed their vulnerability of getting seriously injured lower to that of their peers. Such a tendency has also been widely observed for a variety of events in the field of driving (Harré, Susan, \& O'Neill, 2005), in crime (Perloff \& Fetzer, 1986), and in health (Weinstein, 1980). A distinction must be established between dispositional optimism (Scheier \& Carver, 1985) and 
specific domain-related comparative optimism. While dispositional optimism is defined as a personality trait, or a generalized positive expectancy to experience positive outcomes, domain-related comparative optimism involves rating one's own risk relative to that of the average peer and can vary from one event to another. Thus, comparative optimism examined within a specific context may have no link with optimism measured as a general personality trait (Davidson \& Prkachin, 1997; Martha el al., 2009; Radcliffe \& Klein, 2002).

As people's perceived comparative risks may be related to the way people perceive safety recommendations (Chappé, Verlhiac, \& Meyer, 2007; Perloff \& Fetzer, 1986) and adopt cautious behaviors (Klein, 1997; McKenna, Stanier, \& Lewis, 1991), it seems important to focus on how risk sports practitioners, in comparison with others, perceive their risk exposure whilst participating in their activity. This was the purpose of the present study.

We investigated two types of risk sports practitioners: high-risk sportsmen (i.e., skydivers, BASE-jumpers, and paragliders) and moderate-risk sportsmen (i.e., triathletes). We had two objectives. The first was to examine how risk sports practitioners perceive their personal abilities to manage risks (AMR) and how they perceive their AMR in comparison to that of a specific referent (i.e., the average sportsman participating in the same sport). We also examined how risk sports practitioners assess their vulnerability to a serious injury (VSI) whilst participating in comparison to (a) a specific referent and (b) a non-specific referent (i.e., the average sportsman).

In the field of road traffic (Armor \& Taylor, 1998; Causse, Delhomme, \& Kouabenan, 2005), as well as that of high-risk sports such as rock climbing (Martha et al., 2009), studies have shown that comparative optimism may be not systematic, as respondents may perceive their own VSI or their own AMR as similar to those of others. Respondents may also express comparative pessimism, perceiving their own VSI as greater, or their own AMR as lower, than that of others, particularly when they have experienced negative events such as accidents (Rutter, Quine, \& Albery, 1998) or when they feel they have little personal control over dangers (Harris, 1996). Since the present study focused on sports that involve varying degrees of risk, which remain uncontrollable, we might expect that comparative optimism will not be prevalent amongst risk sports practitioners. More precisely, we hypothesized that risk sports practitioners would perceive their AMR and their VSI as being similar to those of the specific referent (Hypothesis 1) and that they would express comparative pessimism when they compare their VSI with that of the nonspecific referent (Hypothesis 2). We hypothesized that they would imagine the nonspecific referent as the typical sportsman who does not necessarily practice a dangerous sport.

The second objective of this study was to examine which variables influenced risk sports practitioners' perceived comparative VSI. We first considered the role of age and sporting experience (e.g., frequency of participation) on perceived comparative VSI. Then, we examined the role of perceived personal AMR. Perceived personal AMR has been negatively linked to perceived comparative vulnerability in the field of highrisk sports (Moen \& Rundmo, 2005), as well as in driving (Delhomme, 1991). In this vein, we expected that perceived personal AMR will be negatively linked to perceived 
comparative VSI (Hypothesis 3). We also aimed to examine the role of the motive of playing to the limit on perceived comparative VSI. Based on the hypothesis that risk sport practitioners' perceived comparative risks would be in some way realistic, we expected that participants who like playing to the limit will be conscious of the risk they take and thus will be likely to assess themselves as being more exposed to the risk of injury than the average sportsman (Hypothesis 4). Finally, in line with studies (e.g., Rutter et al., 1998) who found a positive link between accident history and an increased perceived vulnerability, we expected that past injury episodes will be positively linked with perceived comparative VSI (Hypothesis 5).

\section{Method}

\section{PARTICIPANTS}

This study was approved by the local university ethics committee of the University of the Mediterranean in Marseilles, France. We limited the analysis to men because there were very few women who responded to the surveys. Although risk sport participation does not guarantee injury or death and there are numerous ways to participate in most sports in relative safety (Donnelly, 2004), risk of serious injury or death is a ubiquitous feature of some sporting experiences. This is the case of skydiving, BASE-jumping, and paragliding, which we qualified as high-risk sports in this study. In order to determine the risk of danger in these sporting activities, we have quantified the risk of activity-related injury or death based on the number of injuries or deaths per 1,000 participants per year (Spinks \& McClure, 2007). According to the French federations of paragliding and skydiving, each of these sports has claimed about 12 lives per year in France over the past 5 years-that is to say 1 death per 3,500 skydivers and per 2,580 paragliders. BASEjumping, a sport in which participants use a parachute to jump from fixed objects le.g., buildings, bridges, and cliffs; see Cooper \& Laurendeau, 2007), is also a high-risk sport. This sport has resulted in 1 death per year in France amongst the 200 French BASEjumpers over the past 5 years (Di Giovanni, 2007). In contrast to those high-risk sports, other sports, such as dancing, fitness, or swimming, can be classified in the category of low-risk sports as they involve a very low probability of participants being seriously injured or killed. Finally, at an intermediate level, moderate-risk sports are those which usually do not result in fatalities, such as in high-risk sports, but are more likely to involve physical accidents compared with low-risk sport (Zuckerman, 1983)-for example the triathlon, which involves risk of collision or falling whilst cycling. According to the French federation of triathlon, this sport has caused about 90 physical injuries per year over the past 5 years (i.e., 1 injury per 233 participants).

Though 462 male adults gave their informed consent and took part to the survey, the analysis was based on data from 432 respondents since we removed 30 incomplete questionnaires. The sample was composed of 313 high-risk sportsmen $(73$ paragliders, 39 BASE-jumpers, and 201 skydivers), and 119 moderate-risk sportsmen (triathletes). Further details about response rate and participants' characteristics are provided in the procedure section and in the results section, respectively. 


\section{MATERIAL}

The questionnaire consisted of four sections. Formulation of the items was subgroup specific, since specific terms were used to designate the sporting activity as well as the participants involved in each activity (e.g., the terms skydiving and skydivers were used in the questionnaire addressed to the skydivers). For the purposes of illustration, we present the items that concerned the skydivers.

The first section gathered general information on variables such as age and sporting experience, including number of years of participation, frequency of participation, and injury episodes having necessitated medical attention over the past 3 years whilst participating. Frequency of participation was measured by means of different criteria for each of the groups. For skydivers, BASE-jumpers, and paragliders, we measured the number of jumps or flights per year, which is considered by these sportsmen to be a good indication of frequency (Laurendeau, 2006; Martha \& Griffet, 2006). For triathletes, we measured frequency of participation by asking the number of times per week they train for triathlon. We then converted weekly participation to yearly participation in order to compare frequency of participation between the four groups.

In the second section, three items developed for the need of the present survey measured perceived personal AMR (Cronbach's alpha $=.78$; i.e., "Whilst skydiving, I think that most of the outcomes are under my control," "I think that my know-how in participating in skydiving safely is high," "Whilst skydiving, I feel myself able to manage most of the risks"). Responses were given on a 7-point likert scale as recommended by Diefenbach, Weinstein, and O'Reilly (1993) in their study on appropriate measures for assessing perceptions of susceptibility to health and safety risks; responses ranged from 1 (I strongly disagree) to 7 (/ strongly agree).

In the third section, perceived comparative AMR and VSI were obtained by the direct method for measuring perceived comparative risks: a single item asking the respondents to compare themselves directly to the average. This use of a single item has been validated in surveys investigating perceived comparative vulnerability and AMR (e.g., Delhomme, 1991; Rutter et al., 1998). We first measured perceived comparative VSI by asking participants to compare themselves to both a specific (Item 1 ) and a nospecific referent (Item 2): "In your opinion, what is your probability of being seriously injured whilst skydiving in comparison to that of the average same-age and same-sex skydiver?" (Item 1), and "In your opinion, what is your probability of being seriously injured in your sport in comparison to that of the average same-age and same-sex sportsperson in his sport?" (ltem 2). Participants answered on a scale ranging from -3 (much less likely) to +3 (much more likely). Thus, scores lower than 0 corresponded with comparative optimism, and scores higher than 0 corresponded with comparative pessimism. Scores close to 0 indicated that participants rateed their VSI as being similar to that of the aforementioned referents. We then measured perceived comparative AMR by asking respondents to compare themselves to the specific referent (ltem 3 ) by answering the following: "In your opinion, how able are you to manage risks inherent to skydiving in comparison to the average same-age and same-sex skydiver?" (Item 3). Participants' 
responses were given on a scale ranging from -3 (much worse) to +3 (much better). Thus, scores lower than 0 corresponded with comparative pessimism, and scores higher than 0 corresponded with comparative optimism. Scores close to zero indicated that participants rated their AMR as being similar to that of the specific referent.

In the fourth and final section, we used the motives for sport participation scale (Recours, Souville, \& Griffet, 2004), which originally contained 13 items measuring four subscales: exhibitionism, competition, sociability, and playing to the limit. For the purpose of the present survey, we only used the four-item playing to the limit subscale (in the present survey, Cronbach's alpha $=.74)$. One example of these items was the following: "What I like in skydiving is the action close to the breaking point." Answers were given on a scale ranging from 1 (/ strongly disagree) to 7 (/ strongly agree).

\section{Procedure}

Participants were contacted through mail, e-mail, clubs, and other practice sites. We first contacted BASE-jumpers by way of e-mail, using e-mail addresses obtained from the website of the French base association (http://www.base-jump.com). The response rate amongst BASE-jumpers was the lowest in our study since $28(49 \%)$ of the 57 BASEjumpers we contacted gave their informed consent to take part in the survey. The rest refused to participate (17\%), due to lack of time, lack of motivation, or they did not reply $(34 \%)$. In addition, 12 BASE-jumpers were invited to take part in the survey in the field and 11 agreed to participate.

Skydivers and paragliders were also contacted by email: the French federations of paragliding and skydiving provided email addresses of their participant members. We contacted 200 skydivers and 75 paragliders. Response rates were high with 152 skydivers $(76 \%)$ and 53 paragliders $(71 \%)$ agreeing to participate. Then, the participation of 22 additional skydivers on their drop zone joined the study from an oral invitation made by the director of the drop zone. As we did not know how many skydivers were present at the drop zone at this time, response rate was difficult to quantify in this case. We also invited 26 additional paragliders on their practice sites to take part in the survey. Amongst them, $77 \%(n=20)$ agreed to fill out the questionnaire.

We contacted triathletes through a club in Marseilles. We negotiated access to the club with the assistance of colleagues who worked there and then asked the triathletes if they were willing to participate by means of verbal announcements. Again, response rate was difficult to quantify since we did not know how many participants were present each time we followed this procedure. Amongst the triathletes who were present, 126 were willing to participate and provided their address. We contacted them at a later date to complete the questionnaire.

We told the participants that we wanted to conduct a study on the theme of sporting experience and asked them to consent to fill out a questionnaire. We stressed to participants that their responses were anonymous, that participants in several sports took part in the survey, and that it was important for us to receive honest and accurate information. While some participants asked us to send the questionnaire by mail and 
subsequently returned it anonymously in a stamped addressed envelope, many filled out the questionnaires on-site (e.g., at a skydiving drop zone) and deposited them in a large box containing others' questionnaires to preserve respondents' anonymity.

\section{Statistical Analyses}

Pearson correlations were calculated to identify the relations among perceived personal $A M R$, playing to the limit, perceived comparative VSI, perceived comparative AMR, and injury episodes.

One-sample - tests were carried out to measure whether participants expressed comparative pessimism, comparative optimism, or whether they perceived their AMR and VSI as being similar to those of the nonspecific and specific referents (i.e., whether their perceived comparative VSI and AMR scores were higher, lower, or close to zero).

We compared the four sporting activities with regard to several dependent variables (perceived personal AMR, playing to the limit, perceived comparative VSI, and perceived comparative AMR). For this purpose, we conducted a multivariate analysis of variance (MANOVA). Then, univariate analyses (ANOVAs) were carried out to test which dependent variables were responsible for the differences in mean vectors that were shown in the MANOVA. Seeing that increased experience may play a role in perceived comparative risks (e.g., Laurendeau, 2006; Lois, 2001), number of years of participation was entered as a covariate. The $\eta^{2}$ values were used to control for the effect size of both sporting activity and number of years of participation. ANOVAs were supplemented by pairwise comparisons with Tukey-Kramer test, recommended for the situation of unequal sample sizes (Toothacker, 1993) to determine differences between groups.

Finally, hierarchical regression analyses were performed to examine factors predicting perceived comparative VSI in comparison with that of the nonspecific referent. We did not aim to predict other variables, such as perceived comparative AMR or perceived comparative VSI in comparison with those of the specific referent, since participants expressed no significant optimism nor pessimism when making such comparative judgements. Since ANOVAs revealed a difference between the four groups on perceived comparative VSI and the nonspecific referent, we analysed the factors predicting this dependent variable amongst each group of sportsmen separately.

First, we examined whether age and sporting characteristics predicted variance in perceived comparative VSI (Step 1) without considering the role of the psychosocial variables correlated to perceived comparative VSI, which may have attenuated the effects of age and sporting characteristics. Then we entered psychosocial variables in the model (Step 2): perceived personal AMR and playing to the limit. Since we expected that the link found in correlational analyses between past injury episodes and perceived comparative VSI might mask the effects of the other variables, we examined the role of past injury episodes (Step 3). This also allowed us to observe whether any associations remained significant after accounting for past injury episodes. 


\section{Results}

Table 1 provides the distribution of the sample, as well as the respondents' average age and sporting characteristics, including past injury episodes. There were significant differences between the four groups in mean age, $F(3,431)=8.6, p<.001$, and in number of years of participation, $F(3,429)=9.4, p<.001$, as BASE-jumpers were younger than the three other groups $(p<.001)$ and as skydivers had a higher number of years of participation than had the three other groups $(p<.001)$. There was a difference between the four groups in frequency of participation, $F(3,429)=36.2, p<.001$. Triathletes had a higher frequency of participation than the other three groups $(p<.001)$, as well as skydivers, compared with paragliders $(p<.001)$ and BASE-jumpers $(p<.001)$. It should be noted, however, that the units measuring frequency of participation were not consistent across the sports, due to different technical elements of the activities. The percentage of respondents reporting having been injured at least once over the last 3 years was lower amongst the triathletes than amongst the other groups, $\chi^{2}(3)=14.3, p<.001, \phi=.18$.

Correlations amongst the measures are shown in Table 2. The pattern of correlations between the study variables was nearly the same amongst the four groups of sportsmen, except for the motive of playing to the limit. Amongst BASE-jumpers, paragliders, and skydivers, playing to the limit was related to perceived personal AMR $(r>0.28$, $p<.01)$ and perceived VSI compared with the nonspecific referents $(r>.30, p<.01)$; these relationships were not significant amongst triathletes. Amongst all the groups, the association between perceived personal AMR and perceived comparative AMR was small $r<.17, p<.05)$, while the inverse association between perceived AMR and perceived VSI compared with the specific referents was high $(r<-.61, p<.001)$. Moderate positive associations were found between past injury episodes and perceived comparative VSI $(r>.28, p<.01)$. Past injury episodes were also negatively related to perceived personal AMR amongst all the groups $(r<-.19, p<.05)$.

Table 1. Participants' Characteristics Regarding Age and Sporting Activity $(n=432)$

\begin{tabular}{l|c|c|c|c} 
& $\begin{array}{c}\text { BASE-jumpers } \\
(n=39)\end{array}$ & $\begin{array}{c}\text { Paragliders } \\
(n=73)\end{array}$ & $\begin{array}{c}\text { Skydivers } \\
(n=201)\end{array}$ & $\begin{array}{c}\text { Triathletes } \\
(n=119)\end{array}$ \\
\hline Mean age (SD) & $31.6(5.9)$ & $37.6(11.8)$ & $36.0(9.7)$ & $36.3(8.6)$ \\
\hline $\begin{array}{l}\text { Mean frequency of } \\
\text { participotion (SD) }\end{array}$ & $\begin{array}{c}55.2(12.1) \\
\text { jumps per year }\end{array}$ & $\begin{array}{c}43.1(16.6) \\
\text { flights per year }\end{array}$ & $\begin{array}{c}82.3(14.3) \\
\text { jumps per year }\end{array}$ & $\begin{array}{c}150.8(62.4) \\
\text { times per year }\end{array}$ \\
\hline $\begin{array}{l}\text { Mean number of years having } \\
\text { participated (SD) }\end{array}$ & $3.1(1.4)$ & $4.5(1.9)$ & $6.4(2.0)$ & $4.6(1.4)$ \\
\hline $\begin{array}{l}\text { \% (n) of respondents having } \\
\text { experienced at least one injury } \\
\text { episode (a) }\end{array}$ & $61(24)$ & $55(40)$ & $67(134)$ & $23(27)$ \\
\hline $\begin{array}{l}\text { Injury episodes (b): Median } \\
\text { (first, third quartile) }\end{array}$ & $1.0(0.0,2.0)$ & $1.0(0.0,1.0)$ & $1.0(0.0,2.0)$ & $0.0(0.0,0.0)$ \\
\hline
\end{tabular}

Note: a Injury episodes necessitating medical attention experienced over the last 3 years while participating.

b Since data on injury episodes are skewed, medians and interquartile ranges are provided instead of means (SDS) 
Table 2. Correlations Between Perceived Personal AMR, Motive of Playing to the Limit, Perceived Comparative AMR, Perceived Comparative VSI, and Past Injury Episodes Amongst Each Group of Participants

\begin{tabular}{|c|c|c|c|c|c|c|}
\hline & & 1 & 2 & 3 & 4 & 5 \\
\hline \multirow{4}{*}{ 1. Perceived personal AMR } & BASE-jumpers & _ & & & & \\
\hline & Pasagliders & - & & & & \\
\hline & Skydivers & - & & & & \\
\hline & Triothletes & - & & & & \\
\hline \multirow{4}{*}{ 2. Motive of ploying to the limit } & BASE-jumpers & $.31^{* *}$ & _ & & & \\
\hline & Paragliders & $.29 * *$ & _ & & & \\
\hline & Skydivers & $.28^{* *}$ & - & & & \\
\hline & Triathletes & .09 & _ & & & \\
\hline \multirow{4}{*}{$\begin{array}{l}\text { 3. Perceived AMR in comparison with } \\
\text { that of the specific referent }\end{array}$} & BASE-jumpers & $.15^{*}$ & .14 & - & & \\
\hline & Parogliders & $.16^{*}$ & $.15^{*}$ & _- & & \\
\hline & Skydivers & $.17^{*}$ & .14 & - & & \\
\hline & Triothletes & $.15^{*}$ & .12 & - & & \\
\hline \multirow{4}{*}{$\begin{array}{l}\text { 4. Perceived VSI in comporison with } \\
\text { that of the specific referent }{ }^{\circ}\end{array}$} & BASE-jumpers & $-.15^{*}$ & $.17^{*}$ & $-.61^{* * * *}$ & - & \\
\hline & Paragliders & -.12 & $.20^{*}$ & $-.64^{* * *}$ & _ & \\
\hline & Skydivers & $-.14^{*}$ & $.22^{*}$ & $-.66^{* * *}$ & - & \\
\hline & Triathletes & $-.19^{*}$ & .10 & $-.62^{* * *}$ & _- & \\
\hline \multirow{4}{*}{$\begin{array}{l}\text { 5. Perceived VSI in comparison with } \\
\text { that of the non-sperific referent }{ }^{b}\end{array}$} & BASE-jumpers & $-.30^{* *}$ & $.35^{* *}$ & $-.18^{*}$ & $.20^{*}$ & - \\
\hline & Paragliders & $-.31^{* *}$ & $.32^{* *}$ & $-.19^{*}$ & $.21^{*}$ & _ \\
\hline & Skydivers & $-.34^{* *}$ & $.30^{* *}$ & $-.17^{*}$ & $.22^{*}$ & _ \\
\hline & Triothletes & $-.31^{* *}$ & .11 & $-.16^{*}$ & $.19^{*}$ & _- \\
\hline \multirow{4}{*}{ 6. Past injury episodes } & BASE-jumpers & $-.27^{* *}$ & $-.20^{*}$ & $-.15^{*}$ & $.35^{* *}$ & $.42^{* *}$ \\
\hline & Paragliders & $-.25^{* *}$ & $-.21^{*}$ & $-.16^{*}$ & $.34^{* *}$ & $.40^{* *}$ \\
\hline & Skydivers & $-.25^{* *}$ & $-.22^{*}$ & $-.17^{*}$ & $.36^{* *}$ & $.40^{* *}$ \\
\hline & Triothletes & $-.19^{*}$ & -.13 & $-.15^{*}$ & $.28^{* *}$ & $.31^{* *}$ \\
\hline
\end{tabular}

Note: * AMR = abilities to manage risks inherent to the sporting activity. VSI = vulnerability to a serious injury whilst participating. " The specific referent was the average same-age and same-sex sportsman participating in the same sport. 'The nonspecific referent was the averoge same-age and same-sex sportsman. $p<.05$ (2-tailed), ${ }^{* *} p<.01$ (2-tailed), ${ }^{* * *} p<.001$ (2-tailed). 
Descriptive statistics and statistical differences according to the group in perceived personal AMR, motive of playing to the limit, and perceived comparative AMR and VSI are provided in Table 3. MANOVA revealed significant group differences on the dependent variables, Wilks's $\Lambda=0.56, F(15,1703)=3.42, p<.001$. There was a difference between the four groups on perceived personal AMR, $F(3,430)=7.9, p<.001, \eta^{2}=.11$, controlling for number of years of participation which had no effect, $F(1,429)=1.9$, $p=.09$, as triathletes reported higher scores on perceived personal AMR than did skydivers $(p=.009)$, BASE-jumpers $(p<.001)$, and paragliders $(p<.001)$. There was also a difference between the groups on the motive of playing to the limit $F(3,429)=8.8$, $p<.001, \eta^{2}=.10$; controlling for number of years of participation had no effect, $F(1$, $429)=.8, p=.32$, since triathletes reported higher scores on this motive than the three groups of high-risk sports practitioners $(p<.001)$. The four groups of sportsmen perceived their AMR and their VSI as being similar to those of the specific referent. When the comparison target was the nonspecific referent, perceived comparative VSI was different amongst the four groups $F(3,430)=7.72, p<.001, \eta^{2}=.12$; controlling for number of years of participation had a positive effect, $F(1,429)=3.8, p<.05, \eta^{2}=.05$. Only triathletes expressed comparative optimism, scoring -1.11 on a scale that ran from -3 to $+3(p<.001)$. Skydivers, BASE-jumpers, and paragliders expressed comparative pessimism, scoring more than $.30(p<.01)$.

Hierarchical regression analyses (see Table 4 ) were performed to examine factors related to perceived VSI compared with the nonspecific referent (i.e., the average same-age and same-sex sportsman) amongst each group of sportsmen. In the first step, the variables age, number of years of participation, and frequency of participation accounted for between $10 \%$ and $14 \%$ of the variance (adjusted $R^{\prime}$ ), $F \geq 4.53, p<.01$. Number of years of participation was positively associated with perceived comparative VSI amongst the four groups, $(\beta \geq .19, p<.01)$, as was age amongst paragliders, skydivers, and triathletes $(\beta \geq .14, p<.05)$, and as was frequency of participation amongst BASE-jumpers, paragliders, and skydivers $(\beta \geq .13, p<.05)$.

In Step 2, perceived personal AMR and playing to the limit explained between $4 \%$ and $5 \%$ of additional variance $\left(\Delta R^{2} \geq 0.15, F \geq 5.31, p<.01\right)$. Playing to the limit served as a predictor for perceived comparative VSI amongst the three groups of highrisk sportsmen $(\beta \geq .24, p<.01)$, as did age amongst paragliders, skydivers, and triathletes $(\beta \geq .12, p<.05)$. Perceived personal AMR was an inverse predictor of perceived comparative VSI amongst the four groups $(\beta \leq-.16, p<.05)$.

In Step 3, injury experience explained between $3 \%$ and $10 \%$ of additional variance $\left(\Delta R^{2} \geq 0.23, F \geq 6.91, p<.001\right)$. Injury experience served as a strong predictor for perceived comparative VSI amongst the three groups of high-risk sportsmen $(\beta \geq 0.28$, $p<.01$ ), while it was a slight but significant predictor amongst triathletes $\beta=.12$, $p<.05)$. Playing to the limit remained positively associated with perceived comparative VSI amongst the three groups of high-risk sportsmen $(\beta \geq .21, p<.01)$, as did number of years of participation amongst the four groups $(\beta \geq .15, p<.01)$ and frequency of participation amongst the BASE-jumpers, the paragliders, and the skydivers $(\beta \geq .11$, $p<.05)$. Perceived personal AMR remained a significant inverse predictor of perceived 
Table 3. Means, Standard Deviations, and Group Differences in Perceived Personal AMR, Motive of Playing to the Limit, Perceived Comparative AMR, and VSI $(N=432)$

\begin{tabular}{|c|c|c|c|c|c|c|}
\hline Variables & Groups & $n$ & - & SD & $t$ (p volue) & $\begin{array}{c}F \text {-value (p value) and } \\
\text { Tukey-Kromer post-hoc } \\
\text { tests ( } p \text { value) on the } \\
\text { four groups }\end{array}$ \\
\hline \multirow{4}{*}{ Perceived personal AMR } & BASE-jumpers & 39 & 2.1 & 1.2 & - & \multirow{4}{*}{$\begin{array}{l}A(3,430)=7.9(p<.001) \\
\text { Triathletes }>\text { BASE-jumpers, } \\
\text { Paragliders }(p<.001) \\
\text { Triathletes }>\text { Skydivers } \\
(p=.009)\end{array}$} \\
\hline & Paragliders & 73 & 2.4 & 1.3 & - & \\
\hline & Skydivers & 201 & 2.9 & 1.4 & - & \\
\hline & Triothletes & 119 & 4.1 & 1.0 & - & \\
\hline \multirow{4}{*}{ Playing to the limit } & BASE-jumpers & 39 & 3.8 & 1.4 & - & \multirow{4}{*}{$\begin{array}{l}F(3,429)=8.8(p<.001) \\
\text { Triathletes }>\text { BASE-jumpers, } \\
\text { Paragliders, Skydivers } \\
(p<.001)\end{array}$} \\
\hline & Poragliders & 73 & 3.6 & 1.2 & - & \\
\hline & Skydivers & 201 & 3.4 & 1.1 & - & \\
\hline & Triathletes & 119 & 4.5 & 1.2 & - & \\
\hline \multirow{4}{*}{$\begin{array}{l}\text { Perceived AMR in } \\
\text { comporison with that of } \\
\text { the specilic referent" }\end{array}$} & BASE-jumpers & 39 & .09 & 1.0 & $1.1(p=.132)$ & \multirow{4}{*}{$F(3,430)=1.64(p=.234)$} \\
\hline & Paragliders & 73 & .12 & .9 & $1.3(p=.101)$ & \\
\hline & Skydivers & 201 & .11 & 1.0 & $1.2(p=.115)$ & \\
\hline & Triathletes & 119 & .08 & .8 & $1.0(p=.202)$ & \\
\hline \multirow{4}{*}{$\begin{array}{l}\text { Perceived VSI in } \\
\text { comporison with that of } \\
\text { the specific referent }\end{array}$} & BASE-jumpers & 39 & -.10 & .7 & $1.1(p=.133)$ & \multirow{4}{*}{$f(3,430)=1.78(p=.145)$} \\
\hline & Paragliders & 73 & -.11 & .8 & $1.2(p=.110)$ & \\
\hline & Skydivers & 201 & -.12 & .7 & $1.2(p=.102)$ & \\
\hline & Triathleles & 119 & -.13 & .9 & $1.3(p=.088)$ & \\
\hline \multirow{4}{*}{$\begin{array}{l}\text { Perceived VSI in } \\
\text { comparison with that } \\
\text { of the non-specific } \\
\text { referent' }\end{array}$} & BASE-jumpers & 39 & .45 & 1.2 & $4.6(p<.001)$ & \multirow{4}{*}{$\begin{array}{l}F(3,430)=7.72(p<.001) \\
\text { Triothletes }<\text { BASE-jumpers, } \\
\text { Paragliders, Skydivers } \\
(p<.001)\end{array}$} \\
\hline & Parogliders & 73 & .38 & 1.0 & $3.7(p=.007)$ & \\
\hline & Skydivers & 201 & .30 & .9 & $3.2(p=.009)$ & \\
\hline & Triathletes & 119 & -1.11 & 1.0 & $6.5(p<.001)$ & \\
\hline
\end{tabular}

Note: $t=$ one-somple $t$ test value against 0 . AMR = abilities to manage risks inherent to the sporting activity. VSI = vulnerability to 0 serious injury whilst participating. ${ }^{\circ}$ The specific referent was the average same-age and same-sex sportsman participating in the same sport. ' The nonspecific referent was the average same-age and same-sex sportsman. Possible range for perceived comparative AMR and VSI was -3 to +3 .

comparative VSI $(\beta \leq-.12, p<.01)$. Age remained positively related to perceived comparative VSI amongst the triathlete group only $(\beta=.13, p<.05)$. 
Table 4. Hierarchical Regression Analysis for Variables Predicting Perceived Comparative VSI in Comparison With That of the Nonspecific Referent (i.e., the Average Same-Age and Same-Sex Sportsman), Amongst Each Group of Participants

\begin{tabular}{|c|c|c|c|c|}
\hline & $\begin{array}{c}\text { BASE-jumpers } \\
(n=39)\end{array}$ & $\begin{array}{c}\text { Paragliders } \\
(n=73)\end{array}$ & $\begin{array}{l}\text { Skydivers } \\
(n=201)\end{array}$ & $\begin{array}{l}\text { Triathletes } \\
(n=119)\end{array}$ \\
\hline Step 1 & 4 & 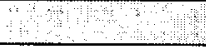 & Win & 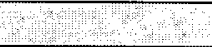 \\
\hline Age & $.10(-.02, .19)$ & $.14^{*}(.04, .30)$ & $.15^{*}(.03, .31)$ & $.18 *(.07, .29)$ \\
\hline $\begin{array}{l}\text { Number of years of } \\
\text { participation }\end{array}$ & $.20^{* *}(.05, .30)$ & $.22^{* *}(.05, .41)$ & $.24^{* *}(.11, .39)$ & $.19^{* *}(.06, .32)$ \\
\hline Frequency of porticipotion & $.13 *(.04, .28)$ & $.14^{*}(.03, .28)$ & $.14^{*}(.05, .26)$ & $.10(.02, .21)$ \\
\hline$R^{2}$ & .12 & .14 & .15 & .16 \\
\hline Adjusted $\boldsymbol{R}^{2}$ & .10 & .12 & .12 & .14 \\
\hline Step 2 & 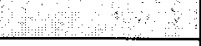 & 4 & 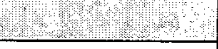 & 19 \\
\hline Age & $.09(-0.01, .21\}$ & $.12 *(.04, .31)$ & $.13^{*}(.03, .27)$ & $.16 *(.03, .30)$ \\
\hline $\begin{array}{l}\text { Number of years of } \\
\text { porticipation }\end{array}$ & $.18^{* *}(.04, .29)$ & $.21^{* *}(.04, .30\}$ & $.20^{* *}(.11, .37)$ & $.17^{* *}(.06, .32)$ \\
\hline Frequency of participation & $.12 *(.05, .29)$ & $.13^{*}(.04, .29)$ & $.12 *(.06, .29)$ & $.09(.01, .21)$ \\
\hline Perceived personal AMR & $-.16^{*}(-.31,-.02)$ & $-.18^{* *}(-.33,-.01)$ & $-.20^{* * *}(-.35,-.04)$ & $-.24^{* * *}(-.41,-.07)$ \\
\hline Playing to the limit & $.32 * *(.14, .45)$ & $.24^{* * *}(.08, .35)$ & $.24^{* * *}(.07, .35)$ & $.09(-.05, .18)$ \\
\hline Adjusted $R^{2}$ & 0.15 & 0.17 & 0.16 & 0.18 \\
\hline Siep 3 & 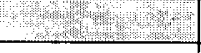 & Pat & 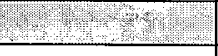 & 3. \\
\hline Age & $.04(-.04, .13)$ & $-.02(-.10, .06)$ & $-.06(-.18, .03)$ & $.13^{*}(.01, .24)$ \\
\hline $\begin{array}{l}\text { Number of years of } \\
\text { participation }\end{array}$ & $.16^{* *}(.02, .34)$ & $.18^{* *}(.03, .32)$ & $.17^{* *}(.03, .31)$ & $.15^{* *}(.04, .28)$ \\
\hline Frequency of participation & $.11^{*}(.02, .24)$ & $.12^{*}(.03, .26)$ & $.12 *(.03, .27)$ & $.07(-.02, .19)$ \\
\hline Perceived personal AMR & $-.12^{*}(-.22,-.01)$ & $-.14^{* *}(-.30,-.02)$ & $-.16^{* * *}(-.31,-.01)$ & $-.23^{* * *}(-.40,-.08)$ \\
\hline Playing to the limit & $.27^{* *}(.14, .42)$ & $.21^{* * *}(.10, .33)$ & $.22^{* * *}(.11, .34)$ & $.13(.01, .22)$ \\
\hline Injury experience ${ }^{b}$ & $.31^{* *}(.15, .43)$ & $.29^{* *}(.14, .40)$ & $.28 * *(.13,42)$ & $.12 *(.01, .24)$ \\
\hline$R^{2}$ & .24 & .25 & .28 & .23 \\
\hline Adjusted $R^{2}$ & .22 & .22 & .26 & .21 \\
\hline
\end{tabular}

Note: Standardized coefficients (95\% confidence intervals) are reported. ${ }^{\circ} A M R=$ abilities to manage risks. ${ }^{b}$ Past injury episodes necessitating medical altention experienced over the last 3 years while participating, ${ }^{*} p<.05 ;{ }^{* *} p<.01 ;{ }^{* * *} p<.001$.

\section{Discussion}

In this study, we first aimed to describe how risk sports practitioners assess (a) their personal abilities to manage risks (AMR), (b) their AMR compared with a specific referent (i.e., the average sportsman participating the same sport), and (c) their vulnerability to a serious injury (VSI) compared with a specific referent and a nonspecific referent (i.e., the average sportsman). The second objective was to examine which variables influenced perceived comparative VSI. 
High-risk sportsmen's scores on perceived personal AMR were low since these scores were situated on average beyond the middle of the scale. This seems to speak to high-risk sports practitioners acknowledging that, despite their best efforts, they remain highly exposed to uncontrollable risks (e.g., environmental conditions, other participants' behavior). This finding sheds further light on the notion of control in edgework (lyng, 1990). Lyng (1990) highlights that edgeworkers have an illusory sense that they can control the uncontrollable, an idea not supported here. Instead, this suggests that practitioners believe themselves to be in control of many dimensions of their edgework activities but still recognize that they simply cannot manage everything under conditions of uncertainty (see Laurendeau, 2006). This risk acceptation might be constitutive of the culture of risk (Donnelly, 2004), according to which injury and even death "may have become a way of life that is produced and reproduced in sport" (Donnelly, 2004, p. 33). Triathletes had higher levels of perceived personal AMR than the three groups of high-risk sportsmen. However, we must note that triathletes' score of perceived personal AMR was not high but moderate only, its value corresponding to the middle of the scale. It may be that the triathletes accept that risk is a part of their daily training. As they train in traffic, they are subjected to hazards posed by other people's (i.e., drivers') behoviors. Moreover, the presence of many other participants may entail risk and uncertainty, particularly whilst riding in close proximity to one another. In his study on physical risk and injury in cycling, Albert (1999) also found risk to be a constituent of the culture of this sport. Again, this points to the importance of subcultural constructions of risk (Donnelly, 2004).

When participants assessed their AMR and VSI in comparison to those of the specific referent, their scores on perceived comparative AMR and VSI were all positive and all negative, respectively, but these trends were not statistically significant. This suggests that risk sport practitioners perceived their AMR and their VSI as being similar to those of the specific referent, supporting Hypothesis 1. High-risk sportsmen expressed comparative pessimism when comparing their VSI to that of the nonspecific referent. These results support Hypothesis 2 and go hand in hand with previous studies conducted in the field of road traffic (e.9., Causse et al., 2005), illness (McKenna, Warbuton, \& Winwood, 1993; van der Pligt, 1998), or high-risk sports (Martha et al., 2009), which showed that adults' risky behaviors were not systematically related to comparative optimism. It is important to note that this result contradicts the findings of Moen and Rundmo (2005) that showed skydivers expressed comparative optimism regarding their VSI. However in Moen and Rundmo's (2005) study, a lower percentage (44\%) of skydivers experienced at least one skydiving injury compared with our respondents $(67 \%)$. Thus, we may hypothesize that participants' past injury episodes may help to explain why we found that skydivers did not express comparative optimism.

Triathletes assessed their VSI as being lower than that of the nonspecific referent and similar to that of the specific referent. According to Helweg-Larsen and Shepperd (2001), if respondents tend to express less comparative optimism when compared with a close and specific referent than when compared with a distant or ambiguous one, it may be because respondents change their risk estimates for the referent rather than for their personal estimates. The absolute judgements for a close referent would be easier to 
calculate than would the absolute judgment for a large and generalized referent group, such as the average person (Chambers \& Windschitl, 2004). However, this explanation can not be applied to the high-risk sportsmen's perceived VSI compared with that of the nonspecific referent, since high-risk sportsmen expressed comparative pessimism, supporting Hypothesis 2 . We may hypothesize that when the referent is not specific, highrisk sportsmen likely compare themselves with typical sportsmen who do not practice a dangerous sport. Such an explanation would support the idea that high-risk sportsmen's perceived comparative risks are in some way realistic. Further comprehensive research is needed to identify the type of sportspersons with whom high-risk sportsmen tend to compare themselves when the referent is a nonspecific one.

We observed a negative link between perceived comparative VSI and perceived personal AMR amongst all the participants. This result supports Hypothesis 3, as well as the association between risk perception and self-efficacy, which has been observed amongst sports participants. It also supports whether sports participants were engaged in high-risk (e.g., Moen \& Rundmo, 2005) or low-risk (e.g., Kontos, 2004) sports, as well as amongst drivers (Delhomme, 1991). The role of both past injury episodes and the motive of playing to the limits on perceived comparative VSI support Hypothesis 4 and Hypothesis 5. It also lends support to a relative realism of perceived comparative risks amongst high-risk sportsmen.

Although the triathletes reported more motivation for playing to the limit than did the three groups of high-risk sportsmen, this motive was not related to perceived comparative VSI amongst the triathletes. Triathlon involves statistically less risk of serious injury or death than BASE-jumping, paragliding, and skydiving. No triathlon-related fatality has occurred in the last 5 years in France. Thus, playing to the limit whilst training for triathlon probably does not involve as many objective risks as does playing to the limit whilst participating in high-risk sports. This also speaks to the notion that "the edge" is a fluid construction, contingent on the activity as well as on one's perceived risk (lyng 1990).

Finally, our results showed that the more experienced (i.e., more years of participation and more frequent participation) the BASE-jumpers, skydivers, and paragliders, the more exposed to the risk of injury they perceived themselves to be, compared with the nonspecific referent. This may suggest that, when individuals first take up these activities, they express comparative optimism because they do not yet understand the hazards of their sports. After a time in the sport, though, hearing about (and perhaps witnessing) others being hurt or killed, it is more difficult to maintain this optimism. In the case of skydiving, senior jumpers take it upon themselves to coach junior jumpers on making sense of witnessing traumatic events in the sport (Laurendeau, 2006). Zuckerman (1994) reported results different than ours regarding the link between risk experience and risk perception. Taking into account individuals' sensation-seeking trait, he suggested that sensation seeking allows individuals to engage in risk situations that push their comfort zone and elevate their experience level. In return, the more risk experiences sensation seekers acquire, the more comfortable they feel with perceived risk. As lyng (1990) pointed out, though, this level of comfort is indicative of one's cessation of being on the 
edge. As a result, edgeworkers often push themselves, their equipment, and other factors even farther (Laurendeau, 2006). Future studies should examine the role of increased sporting experience in perceived vulnerability using longitudinal protocols and taking into account past experience, coping strategies, and personality traits which may explain the link between risk experience and risk perception.

The findings of the present study should be treated with a degree of caution given the following limitations. First, the cross-sectional research design limits the extent to which we can make claims about causality based on these data. Second, the use of self-reported measures raises concerns about bias in responses. For instance, some of the high-risk sportsmen may have exaggerated their enjoyment of playing to the limit or their perceived risk of getting injured in their sports since there are subcultural norms at play around these issues (see Hunt, 1995; Laurendeau \& Gibbs Van Brunschot, 2006). However, we endeavored to limit response bias by taking precautions when inviting participants to take part in the study, stressing the anonymity of their responses and our interest in receiving honest and accurate information. The response rate may also point toward potential response bias. Perhaps we would have had more reliable information about high-risk sportsmen's risk perception and risk behavior if we could also have obtained participation of high-risk sportsmen who have a propensity to reject psychosociological studies on risk taking. This issue stresses the necessity of taking precautions while inviting high-risk sportsmen to participate in such a study. In this vein, the use of personal and progressive ways of inviting the participants (e.g., after a qualitative study period based only on observation or informal interviews) could be useful. Moreover, our sample only included current participants, a factor which may have influenced the results. Perhaps including participants who were no longer active, above those who were seriously injured, might have involved higher levels of perceived vulnerability. Further research should examine these associations longitudinally.

Third, as we investigated only four types of sporting activities, we must be cautious about generalizing our results to other calegories of sportsmen. This is the case even for other risk sports, as there is some evidence that perceptions of control are not simply a function of belief in a survival instinct, as Lyng suggests (1990). Instead, they are contingent on specific bodies of technical knowledge about particular activities (Laurendeau, 2006). This issue of generalization is further compounded by the fact that our analysis considered only men who participate in these activities. Given that women and men perform edgework differently (Laurendeau, 2008), future studies should explore the experiences of female participants in order to examine potential gender differences in risk perception and risk exposure. This is particularly important in light of Laurendeau's argument (2008) that whether and how women and men engage in edgework is itself part of the process of constructing a particular kind of masculinity or femininity.

Finally, we did not gather qualitative information on respondents' past injury episodes, which could influence perceived comparative VSI. Moreover, we only took into account injuries requiring medical attention, but we could have also measured injuries that involved withdrawal from participation or those seeking treatment or advice from 
nonmedics (Jones, Asghar, \& Llewellyn, 2007). Thus, further research should explore such qualitative and quantitative information in order to better understand the relationship between risk perception and risk exposure.

\section{ConClusion}

In this study, we set out to explore what has been an underresearched area so far in the study of social comparison and risk behaviors, namely, the perceived comparative risks in the field of high-risk sports. High-risk sports participants' tendency to perceive their vulnerability as being similar to that of their peers, and as being higher than that of the nonspecific referent, suggests that practitioners are not oblivious to the hazards of their sports. In a culture of risk (Donnelly, 2004), even participants in risk sports actively engage with the question of how to participate (e.g., what kinds of skydives to do and in what conditions). Contrary to the belief that comparative optimism would be linked to risky behaviors (e.g., Klein, 1997; McKenna et al., 1991), other studies from health (McKenna et al., 1993; Radcliffe \& Klein, 2002; van der Pligt, 1998), driving (Causse et al., 2005), or sport psychology (Martha et al., 2009) literature has shown that comparative optimism is not systematically associated with detrimental behavior and thus may reflect relative accuracy in risk perception. Our results also lend support to a relative realism amongst high-risk sports practitioners, since participants whose behavior puts them at risk are aware of this. Future research is needed to further examine the role that social comparison in general, and perceived comparative risk in particular, play into the risklaking decision-making process.

\section{REFERENGES}

Albert, E. (1999). Dealing with danger: The normalization of risk in cycling. International Review for the Sociology of Sport, 34, 157-171.

Armor, D. A., \& Taylor, S. E., (1998). Situated optimism: Specific outcome expectancies and selfregulation. In M. P. Zanna (Ed.), Advances in experimental social psychology (Vol. 30, pp. 309-379). San Diego: Academic Press.

Bandura, A. (1997). Self-efficacy. The exercise of control. New York: Freeman.

Breivik, G. (1996). Personality, sensation seeking and risk taking among Everest climbers. International Journal of Sport Psychology, 27, 308-320.

Causse, P., Delhomme, P., \& Kouabenan, D. R. (2005). Jugements comparatifs et absolus de deux risques routiers contextualisés et raisons invoquées quant à ces jugements [Absolute and comparative judgements of two situated road risks and reasons evocated for these judgments]. Psychologie du Travail et des Organisations, 11, 191-208.

Celsi, R. L., Rose, R. L., \& Leigh, T. W. (1993). An exploration of the high risk consumption through skydiving. Journal of Consumer Research, 20, 1-23.

Chambers, J. R., \& Windschitl, P. D. (2004). Biases in social comparative judgments: The role of nonmotivated factors in above-average and comparative-optimism effects. Psychological Bul. letin, 130, 813-838.

Chappé, J., Verlhiac, J. F., \& Meyer, T. (2007). Optimisme el pessimisme comparatifs consécutifs à l'exposition à plusieurs messages menaçants [Comparative optimism and pessimism following exposure to several threatening messages]. Revue Européenne de Psychologie Appliquée/ European Review of Applied Psychology, 57, 23-35.

Cooper, J., \& Laurendeav, J. (2007). BASE jumping. In D. Booth and H. Thorpe (Eds.), Berkshire encyclopedia of extreme sports (pp. 20-26). Great Barrington, MA: Berkshire. 
Davidson, K., \& Prkachin, K. (1997). Optimism and unrealistic optimism have an interacting impact on health-promoting behaviour and knowledge changes. Personality and Social Psychology Bulletin, 23, 617-625.

Delhomme, P. (1991). Comparing one's driving with others': Assessment of abilities and frequency of offences. Evidence for a superior conformity of self-bias? Accident Analysis and Prevention, 23, 493-508.

Delle Fave, A., Bassi, M., \& Massimini, F. (2003). Quality of experience and risk perception in high-altitude rock climbing. Journal of Applied Sport Psychology, 15, 82-98.

Demirhan, G. (2005). Mountaineers' risk perception in outdoor-adventure sports: A study of sex and sports experience. Perceptual and Motor Skills, 100, 1155-1160.

Diefenbach, M. A., Weinstein, N. D., \& O'Reilly, J. (1993). Scales for assessing perceptions of health hazard susceptibility. Health Education Research, 8, 181.192.

Di Giovanni, N. (2007). World BASE fatality list. Retrieved October 8, 2007, from http://www. splatula.com/bfl/

Donnelly, P. (2004). Sport and risk culture. In K. Young (Ed.), Sporting bodies, damaged selves: Sociological studies of sports-related injury (pp. 29-58). Boston: Elsevier Press.

Griffet, J. (1994). Décision, risque, émotion [Decision, risk, emotion]. Science et Motricité, 23, 3-12.

Harré, N., Susan, F., \& O'Neill, M. (2005). Self-enhancement, crash-risk optimism and the impact of safety advertisements on young drivers. British Journal of Psychology, 96, 215-230.

Harris, P. (1996). Sufficient grounds for optimism? The relationship between perceived controllability and optimistic bias. Journal of Social and Clinical Psychology, 15, 9-52.

Harris, P., \& Middleton, W. (1994). The illusion of control and optimism about health: On being less at risk but no more in control than others. British Journal of Social Psychology, 33, 369-86.

Helweg-Larsen, M., \& Shepperd, J. A. (2001). Do moderators of the optimistic bias affect personal or target risk estimates? A review of the literature. Personality and Social Psychology Review, 5. 74-95.

Hunt, J. C. (1995). Divers' accounts of normal risk. Symbolic Interaction, 18, 439-462.

Jones, G., Asghar, A., \& Llewellyn, D. J. (2007). The epidemiology of rock climbing injuries. British Journal of Sports Medicine, 42, 773-778.

Klein, W. M. P. (1997). Objective standards are not enough: Affective, self-evaluative, and behavioural responses to social comparison information. Journal of Personality and Social Psychology, 72, 763-774.

Kontos, A. P. (2004). Perceived risk, risk taking, estimation of ability and injury among adolescent sport participants. Journal of Paediatric Psychology, 29, 447.455.

Kusz, K. (2004). Extreme America: The cultural politics of extreme sports in 1990s America. In B. Wheaton (Ed.), Understanding lifestyle sports: Consumption, identity and difference (pp. 197209). London: Routledge.

Laurendeau, J. (2006). "He didn't go in doing a skydive": Sustaining the illusion of control in an edgework activity. Sociological Perspectives, 49, 583-605.

Laurendeau, J. (2008). "Gendered risk regimes": A theoretical consideration of edgework and gender. Sociology of Sport Journal, 25, 293-309.

Laurendeau, J., \& Gibbs Van Brunschot, E. (2006). Policing the edge: Risk and social control in skydiving. Deviant Behavior, 27, 173-201.

Llewellyn, D. J., \& Sanchez, X. (2008). Individual differences and risk taking in rock climbing. Psychology of Sport and Exercise, 9, 413-426.

Llewellyn, D. J., Sanchez, X., Asghar, A., \& Jones, G. (2008). Self-efficacy, risk taking and performance in rock climbing. Personality and Individual Differences, $45,75-81$.

Lois, J. (2001). Peaks and valleys: The gendered emotional culture of edgework. Gender \& Society, $15,381-406$.

Lupton, D. (1999). Risk. London: Routledge.

lyng, S. G. (1990). Edgework: A social psychological analysis of voluntary risk taking. American Journal of Sociology, 95, 851-886.

Lyng, S. G. (Ed.). (2005). Edgework: The sociology of risk faking. New York: Routledge. 
Martha, C., \& Griffet, J. (2006). Le BASE-jump, le jeu le plus sérieux du monde [BASE-jumping: The most serious play in the world]. Ethnologie Française, 36, 635-642.

Martha, C., Sanchez, X., \& Gomà-i-Freixanet (2009). Risk perception as a function of risk exposure amongst rock climbers. Psychology of Sport and Exercise, 10, 193-200.

McKenna, F. P., Stanier, R. A., \& Lewis, C. (1991). Factors underlying illusory self-assessment of driving skill in males and females. Accident Analysis and Prevention, 23, 45-52.

McKenna, F. P., Warbuton, D. M., \& Winwood, M. (1993). Exploring the limits of optimism: The case of smokers' decision making. British Journal of Psychology, 84, 389-394.

Moen, B., \& Rundmo, T. (2005). Predictors of unrealistic optimism: A study of Norwegian risk takers. Journal of Risk Research, 8, 363-382.

Perloff, L. S., \& Fetzer, B. S. (1986). Self-other judgments and perceived vulnerability to victimization. Journal of Personality and Social Psychology, 50, 502-510.

Radcliffe, N. M., \& Klein, W. M. P. (2002). Dispositional, unrealistic, and comparative optimism: Differential relations with the knowledge and processing of risk information and beliefs about personal risk. Personality and Social Psychological Bulletin, 28, 836-846.

Recours, R., Souville, M., \& Griffet, J. (2004). Expressed motives for informal and club/associationbased sports participation: Validation of a questionnaire. Journal of Leisure Research, 36, 1-22.

Rossi, B., \& Cereatti, L. (1993). The sensation seeking in mountain athletes as assessed by Zuckerman's Sensation Seeking Scale. International Journal of Sport Psychology, 24, 417.431.

Rutter, D. R., Quine, L., \& Albery, I. P. (1998). Perceptions of risk in motorcyclists: Unrealistic optimism, relative realism and predictions of behavior. British Journal of Psychology, 89, 681-96.

Scheier, M. F., \& Carver, C. S. (1985). Optimism, coping and health: Assessment and implications of generalized outcome expectancies. Health Psychology, 4, 219-228.

Schneider, T. A., Butryn, T. M., Furst, D. M., \& Masucci, M. A. (2007). A qualitative examination of risk among elite adventure racers. Journal of Sport Behavior, 30, 330-357.

Shepperd, J. A., Carroll, P., Grace, J., \& Meredith, T. (2002). Exploring the causes of comparative optimism. Psychologica Belgica, 42, 65-98.

Slanger, E., \& Rudestam, K. E. (1997). Motivation and dishinibition in high risk sports: Sensation seeking and self-efficacy. Journal of Research in Personality, 31, 355-374.

Spinks, A. B., \& McClure, R. J. (2007). Quantifying the risk of sports injury: A systematic review of activity-specific rates for children under 16 years of age. British Journal of Sport Medicine, 4I, 548-557.

Toothacker, L. E. (1993). Multiple comparisons procedures. Thousand Oaks, CA: Sage.

van der Pligt, J. (1998). Perceived risk and vulnerability as predictors of precautionary behaviour. British Journal of Health Psychology, 3, 1-14.

Weinstein, N. D. (1980). Unrealistic optimism about future life events. Journal of Personality and Social Psychology, 39, 806-820

Young, K. (1993). Violence, risk and liability in male sports culture. Sociology of Sport Journal, 10, 373-396.

Zuckerman, M. (1983). Sensation seeking and sports. Personality and Individual Differences, 4, 285-293.

Zuckerman, M. (1994). Behavioral expressions and biosocial bases of sensation seeking. New York: Cambridge University Press.

\section{ACKNOWLEDGements}

The authors would like to thank the reviewers and the section editor for their helpful comments on this manuscript, and the French triathletes, BASE-jumpers, skydivers, and paragliders for their participation in the study. 
Copyright of International Journal of Sport \& Exercise Psychology is the property of Fitness Information Technology, Inc. and its content may not be copied or emailed to multiple sites or posted to a listserv without the copyright holder's express written permission. However, users may print, download, or email articles for individual use. 